\title{
Improvement of Cu-Pillar Structure Using Advanced
}

\section{Plating Method}

\author{
Jong-Young PARK ${ }^{1,2,3}$, Young-Jae $\mathrm{KIM}^{1,2}$, Joo-Hyong $\mathrm{NOH}^{1,2}$ and Hideo Honma ${ }^{1,2}$ \\ 1. Department of Materials and Surface Engineering, Graduate School of Engineering, Kanto Gakuin University, Ogikubo 1162-2, \\ Odawara 250-0042, Japan \\ 2. Materials and Surface Engineering Research Institute, Kanto Gakuin University, 1162-2 Ogikubo, Odawara 250-0042, Japan \\ 3. Daeduck Electronics Co. Ltd., 335, Somanggongwon-ro, Siheung-si, Gyeonggi-do 15106, Korea
}

\begin{abstract}
The recent appearance of mobile application processor now plays an important role in the semiconductor industry. Additionally, there have been endless demands for small form factor, thin profile, outstanding thermal, mechanical properties and electrical performances in the field of IC packages for mobile application processors. MIS (Molded Interconnect Substrate) can provide ideal and this solution in the mobile industry as it contains multiple solutions for the complicated requirement of the IC packages for application processors. Based on the embedded pattern technology, this solution can provide high I/O count, fine pattern for small form factor, and stable flip chip mounting methods. Other advantages of this solution include stable properties required for high-frequency transmission and high thermal dissipation rate as it is only composed of copper and epoxy mold compound materials. These core techniques for MIS technology can be divided into below major concepts. First, Cu pillar electrolytic plating technology electrically connects the inner and the outer layers using photo-lithography method, instead of laser method. The shape of $\mathrm{Cu}$ pillar and the plating thickness tolerance control are the key parameters. Second, grinding technology is to precisely grind the plated $\mathrm{Cu}$ pillars and applied mold epoxy. And deposition of $\mathrm{Cu}$ layer on top of grinded mold surface can construct fine pattern traces.
\end{abstract}

Key words: Cu-pillar, surface pre-treatment, UV irradiation, plasma etching, plating.

\section{Introduction}

Resent, electric devices have been constant inquiries for small form factor, thin profile, outstanding thermal, mechanical properties and electrical performances in the field of IC packages for mobile application processors [1-3]. MIS (Molded Interconnect Substrate) package technology can provide ideal solutions in the mobile industry as it contains multiple solutions for the complicated requirements of the IC packages for application processors. Based on the embedded pattern technology, MIS can provide high I/O count, fine pattern for small form factor, and stable flip chip mounting methods. Other advantages of this technology include stable properties required for high-frequency transmission and high thermal dissipation rate as it is only composed of copper and

Corresponding author: Jong-Young PARK, Mr, resercher, research fields: materials and surface.
EMC (Epoxy Mold Compound) materials. The biggest advantage of MIS is the ability to achieve ultra-thin thickness by using glass-less material, where the total thickness of a package can be less than $0.1 \mathrm{~mm}[4,5]$. In addition, $\mathrm{Cu}$-pillars for electrical connection between inner and outer layers can be manufactured with photo lithography process followed by grinding process, which replaces laser drilling process, and therefore reduce the production cost. Furthermore, direct formation of traces on top of the mold surface is possible, and it can be applied to Mold first FOWLP (Fan out Wafer Level Package) with I/O count less than 1,000 , along with its excellent degree of freedom in the thickness of package and the traces. Fig. 1 illustrates the representative structure of an MIS product.

In this study, methods to provide the most ideal solutions for each of these three technologies are studied and presented. 


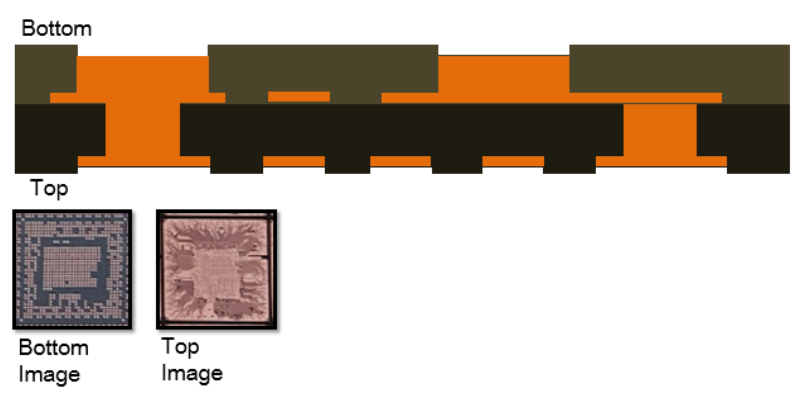

Fig. 1 Structure of MIS technology.

First, $\mathrm{Cu}$ pillar electrolytic plating technology electrically connects the inner and the outer layers use photo-lithography method, instead of laser method. The shape of $\mathrm{Cu}$ pillar and the plating thickness tolerance control are the key parameters. Second, grinding technology grinds precisely the plated $\mathrm{Cu}$ pillars and applied mold epoxy. Last, deposition of $\mathrm{Cu}$ layer on top of grinded mold surface constructs fine pattern traces.

\section{Experimental Setup}

The size of the specimens used in this study includes $100 \mathrm{~mm} \times 100 \mathrm{~mm}$ which can be tested in laboratory based testing environment, as well as $510 \mathrm{~mm} \times 415$ $\mathrm{mm}$, an actual size of the package substrate panel in the PCB manufacturing field. The advantage of testing large size specimens is that it can allow the technology and their results to be immediately and directly applied to the manufacturing field.

\subsection{Cu Pillar Electroplating Technology}

Additives used in the electroplating were DOW Chemical's CP-360, which allows high current density plating ( $\sim 10$ ASD). For this experiment, Haring cell bath, VCP (Vertical Continuous Plating), and the equipments designed to reduce plating thickness variation with optimized distance between anode and cathode (plating specimen) were used [6, 7]. Dry film used in the Photo-Lithography process was HM-40 series by Hitachi, Japan. The aspect ratio was 0.75 , where the plating area was $100 \mu \mathrm{m}$ and the height of the dry film was $75 \mu \mathrm{m}$. Fig. 2 illustrates the structure and design of the plating specimen used in this experiment.

After electroplating, the cross sections of the specimen were analyzed by cutting at the top, middle, and bottom locations, filling with epoxy resin, and polishing with sand paper to observe the surface using optical microscope.

\subsection{Grinding Technology}

After the $\mathrm{Cu}$ pillar formation, the material used for the dielectric layer was mold epoxy from LE series by Ajinomoto, composed of about $20 \%$ phenolic epoxy and $80 \% \mathrm{SiO}_{2}$ by weight. For grinding process, buff type grinder (Teasung, Korea), NC milling type grinder (NC grinding of GPM, Taiwan), and Sand blast type grinder (Elpotec, Japan) were used. The grinding material for the buff type grinder includes ceramic $(\mathrm{SiC})$ and diamond, and the $\mathrm{NC}$ milling type grinder used $\mathrm{SiC}$ grinding material. The sand blast type grinder that can physically etch the specimen by spraying powder type grinding materials at a constant pressure and speed, used grinding material mainly composed of $\mathrm{Al}_{2} \mathrm{O}_{3}$ of about 3-5 um particle size. Fig. 4 displays different types of grinders and their characteristics. The morphology and roughness of the grinded surface were

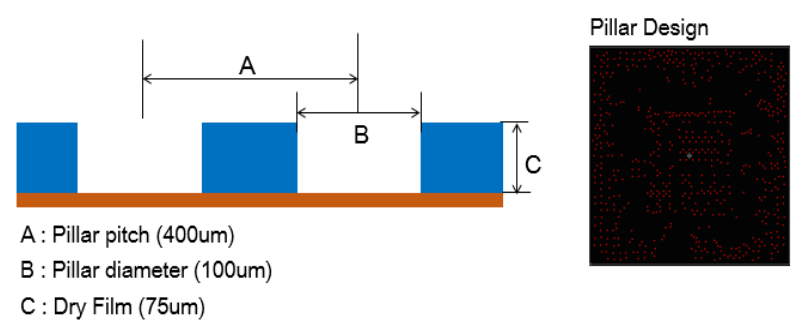

Fig. 2 Design of Cu-pillar for MIS structure.

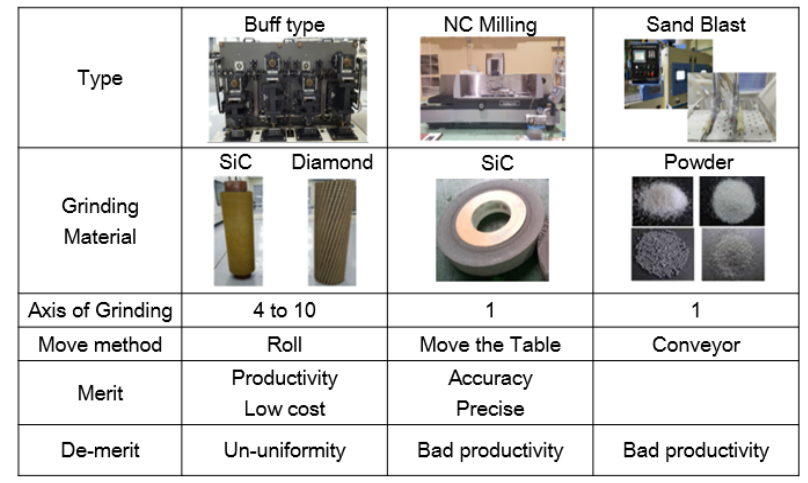

Fig. 3 Feature of each grinding type. 
measured using laser scope and N-SEM, and the cross section was analyzed to confirm if the desired thickness was precisely grinded.

\subsection{Technology of Forming Metal Layer on Top of Grinded Mold Surface}

In order to form metal layer on top of the grinded mold surface, the MV series chemicals by the German company Atotech was used for Permanganate acid treatment for the surface reformation, then electroless $\mathrm{Cu}$ with even thickness was plated without any voids using PEV series by the Japanese company.

\section{Experimental Results}

\subsection{Cu Pillar Electrolytic Plating Technology}

In order to analyze the effect of different type of pre-treatment method prior to the electrolytic plating process, (1) Acid treatment, (2) UV treatment, (3) Plasma treatment, and (4) Descum (Permanganate acid) treatment were applied [8]. Incomplete plating, i.e. skipped plating, occurred when the conventional method of the Acid treatment was used. With plating structures with relatively high aspect ratio, the plating solution does not circulate enough around the Dry Film walls and the exposed plating surfaces, and therefore easily causes air trap which can lead to incomplete plating issues. On the other hand, there was no skipped plating phenomenon with UV, Plasma, and Descum pre-treatment, through which we confirmed certain level of wettability was required for the dry film as well as the plating surface. The level of wettability for different types of pre-treatment was observed with contact angle measurement. Fig. 4 illustrates the level of wettability for different types of pre-treatment. The highest level of wettability was confirmed in the order of UV $>$ Plasma $=$ Descum $>$ Acid pre-treatment methods.

It was confirmed that the plasma treated samples had less dry film undercut issues compared to the other pre-treatment methods. Fig. 5 shows how the dry film undercut is reduced after plasma treatment. We consider that the $\mathrm{CF}_{4}$ gas within Plasma acted as etching factor to the dry film where the undercut was reduced, and that the $\mathrm{N}_{2}$ and $\mathrm{O}_{2}$ gas was ionized and increased the hydrophilicity of the dry film.

As shown in the FT-IR ATR graph of Fig. 6, the peak of Carboxyl group and Methylene group change were detected when treated with UV, Plasma and Descum.

Through the experimental results of different pre-treatment methods, plasma treatment was confirmed to have the most idea results with the plating thickness variation and structure. In perspective of the

\begin{tabular}{|c|c|c|c|c|}
\hline Pre-treatment & Non & Sulfuric acid & $\begin{array}{c}\text { UV } \\
\text { Irradiation }\end{array}$ & Plasma \\
\hline $\begin{array}{c}\text { Active } \\
\text { Element }\end{array}$ & - & Acid & $\mathrm{O}_{3}$ & $\mathrm{~N}_{2}, \mathrm{O}_{2}, \mathrm{CF}_{4}$ \\
\hline \multirow{2}{*}{ Contact angle } & & & & \\
\cline { 2 - 5 } & $69.3^{\circ}$ & $57.1^{\circ}$ & $14.2^{\circ}$ & $21.6^{\circ}$ \\
\hline
\end{tabular}

Fig. 4 Wettability after each pre-treatment.

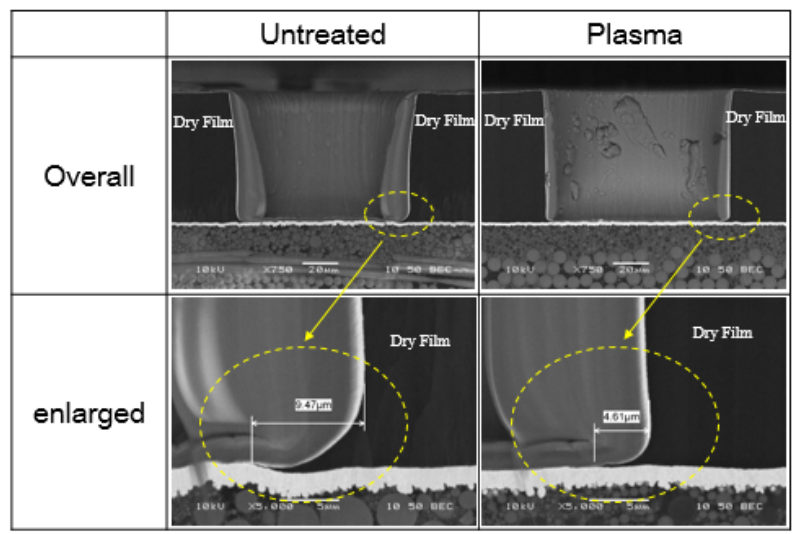

Fig. 5 Observed dry film under-cut after plasma treatment.

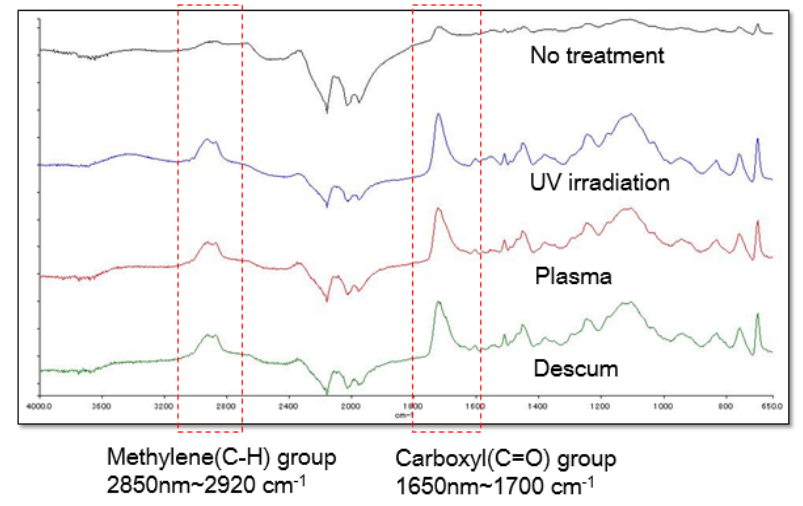

Fig. 6 Observed chemical changes by FT-IR ATR. 
plating facilities, the distance between anode and cathode was optimized to reduce the thickness variation of a relatively large size specimen.

\subsection{Grinding Technology}

After the $\mathrm{Cu}$ pillar formation, mold epoxy was applied and to be grinded with buffer grinder, milling grinding, and sand blast facilities. The buffer grinder uses ceramic and diamond grinders and can grind both sides simultaneously. When ceramic grinder was used, grinding job was done, however, there was significant variation between the center and the edge of the specimen as the grinder expands as rotated. On the other hand, the diamond grinder was able to grind the surface uniformly with its modulus greater than that of $\mathrm{Cu}$ or mold epoxy and relatively small thickness compared with the ceramic grinder. However, it did not seem feasible for fine pattern traces due to its surface roughness and irregularity $[9,10]$. Sand blast method uses small particles of $\mathrm{Al}_{2} \mathrm{O}_{3}$ which was difficult to spray evenly over a large area at a constant speed. In conclusion, sand blast failed to mechanically grind the $\mathrm{Cu}$ structure after it was revealed, therefore the $\mathrm{Cu}$ maintained its height as the epoxy surface was only grinded. It was concluded that with sand blast method, it requires less than $1 \% \mathrm{Cu}$ pillar plating thickness variation, and that it may not be feasible to apply sand blast method. Fig. 7 illustrates the results of different grinding methods.

\subsection{Technology of Forming Metal Layer on Top of Grinded Mold Surface}

Fig. 8 presents the surface morphology and the peel strength between mold epoxy and metal layer for different permanganate treatment time. In case of treated 15 min with permanganate acid gets a high peel strength relatively other treated times.

However, when the 10 to 15 min treated specimen surface appeared to have partial white spot visual inspection. One of probable causes is remained $\mathrm{SiO}_{2}$ particle on the treated surface which EMC material composited above $80 \mathrm{wt} \% \mathrm{SiO}_{2}$ contents. Fig. 9 shows occurred white spot in above 10 min with $\mathrm{MnO}_{4}$ treatment and component of used material.

When the peel strength was measured at the white spot region, relatively low and unstable peel strength was obtained. Low and unstable peel strength can cause delamination of the fine pattern metal traces, therefore cleaning process was reinforced to resolve the white spot issue. Ultrasonic water cleaning that can

\begin{tabular}{|c|c|c|c|c|}
\hline Grinding type & \multicolumn{2}{|c|}{ Buff type } & NC Milling & Sand Blast \\
\hline $\begin{array}{c}\text { Grinding } \\
\text { materials }\end{array}$ & Ceramic & Diamond & $\mathrm{SiC}$ & $\mathrm{Al}_{2} \mathrm{O}_{3}$ \\
\hline $\begin{array}{c}\text { Surface } \\
\text { Morphology }\end{array}$ & & & & \\
\hline Roughness (Rz) & $12.98 \mathrm{um}$ & $13.38 \mathrm{um}$ & $9.91 \mathrm{um}$ & $11.34 \mathrm{um}$ \\
\hline Deviation & $\pm 20 \mathrm{um}$ & $\pm 10 \mathrm{um}$ & $\pm 5 \mathrm{um}$ & $\pm 15 \mathrm{um}$ \\
\hline
\end{tabular}

Fig. 7 Difference according to each grinding types.

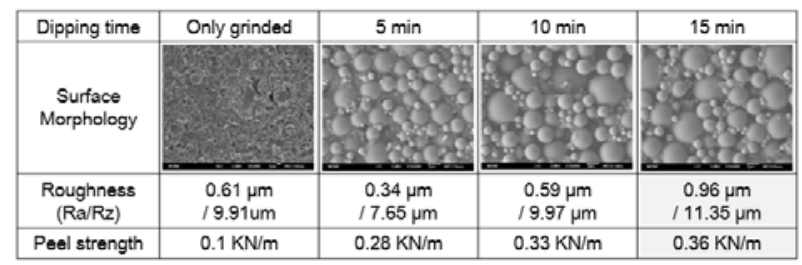

Fig. 8 Observed surface morphology after $\mathrm{MnO}_{4}$ treatment.

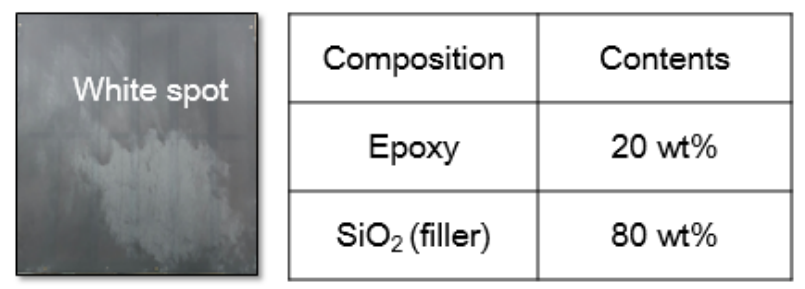

Fig. 9 Observed surface morphology after $\mathrm{MnO}_{4}$ treatment.

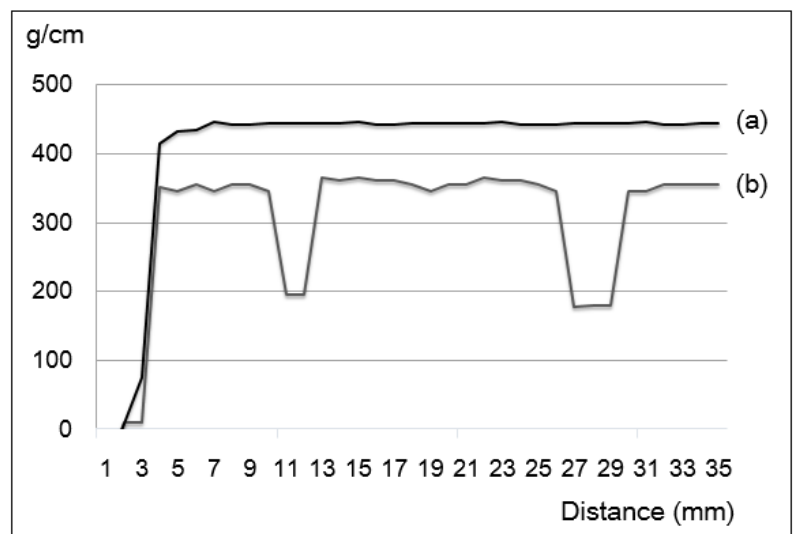

Fig. 10 Peel strength (a) Ultrasonic treated; (b) Untreated. 
apply fine mechanical shock was applied, and effectively removed the $\mathrm{SiO}_{2}$ that remained on the surface with very weak adhesion. The ultrasonic waves were performed from $72 \mathrm{~Hz}$ to $100 \mathrm{~Hz}$ for 5 minutes. Peel strength of the specimen with ultrasonic water cleaning process was higher than that of the specimen without the ultrasonic water cleaning process. Fig. 10 illustrates the effectiveness of the ultrasonic water cleaning procedure after the permanganate treatment.

\section{Conclusions}

MIS (Molded Interconnect Substrate) provides ideal substrate solutions for mobile applications with complicated IC package requirements. Based on the embedded pattern technology, MIS enables fine pattern traces with high $\mathrm{I} / \mathrm{O}$ count and small form factor, providing solid solutions for flip chip mounting processes. MIS is also composed of only copper and mold material therefore provides stable properties for high frequency applications and outstanding thermal properties. For the ideal structures of MIS, $\mathrm{Cu}$ pillar formation process is important as it connects the inner layer to the outer layer. Plasma method was confirmed to be the most ideal pre-treatment method to control the $\mathrm{Cu}$ pillar thickness variation and the flatness of the $\mathrm{Cu}$ plating surface. When plasma pre-treatment was applied, the undercut of the dry film after photo-lithography process was reduced, and the wettability of the surface was enhanced due to surface reformation. The distance between anode and cathode was optimized to reduce the plating thickness variation, however, the optimization of the equipment needs to be studied with more details. For the grinding process after the mold epoxy is applied, milling grinder using $\mathrm{SiC}$ material was confirmed to be the most ideal method to precisely grind the surface to the desired depth. Finally, the grinded mold epoxy surface was treated with permanganate acid, followed by electroless chemical plating. It was found that the permanganate acid treatment of 15 minutes provided the highest peel strength value, and the ultrasonic water cleaning process was effective to remove the white spots on the surface after the permanganate treatment. Especially, alternating the ultrasonic wavelengths between $72 \mathrm{~Hz}$ and $100 \mathrm{~Hz}$ for 5 minutes was very effective in the removal of $\mathrm{SiO}_{2}$ residues on the surface.

In order to produce MIS most ideally, processes of $\mathrm{Cu}$ pillar formation, grinding method, and metal layer formation on top of the grinded surface are very significant. Although positive results and outputs were confirmed through this study, the warpage behavior of during assembly, and the reliability aspects of MIS considering thermal and electrical properties should also be studied in the future.

\section{References}

[1] Zhang, M. et al. 2009. "Research on the Mechanism and the Superficial Microstructure of the Nano-Composited Ceramics under Ultrasonic Polishing." Key Engineering Materials 416: 173-7.

[2] In Lee, Kyoochun Lee, Business Horizons. 2015. 58: 431.

[3] Byoung-uk Kim; ISSUE \& Tech. 2015. 42.

[4] Boyung-Jin, K., et al. 2015. J. Microelectron. Packag. Soc. 22 (2).

[5] Saha, S. K., et al. 2013. Technology Management in the Driven Services (PICMET), 2/44, IEEE.

[6] Doi, T., Mizumoto, K., Tanaka, S., and Yamashita, T. 2004. "Bright Nickel Plating from Nickel Citrate Electroplating Baths." Metal Finishing 102 (4): 26-35.

[7] Lee, S., Hideo, H., and Hideto, Y. 2011. "New Plating Technology.” Kanto Gakuin University Publish: 6.

[8] Young-Jae, K. I. M., Jong-Young, P. A. R. K, Kwan-sun, Y. O. O. N., Joo-Hyong, N. O. H., Hideo, H. O. N. M. A., and Osamu, T. A. K. A. I. 2016. Journal of Nanoscience and Nanotechnology 16 (11): 11186-90.

[9] Sundahl, M., Sikander, E., Ek-Olausson, B., Hjorthage, A., Rosell, L., and Tornevall, M. 1999. "Determinations of PCB within a Project to Develop Cleanup Methods for PCB-Containing Elastic Sealant Used in Outdoor Joints between Concrete Blocks in Buildings." J. of Environ. Monit. 1: 383-7.

[10] Market \& Technology Reports 2016 (Yole development). 